\title{
Aufgaben der studentischen Mitarbeiter und Mitarbeiterinnen
}

\author{
Jasmin Leitner \\ Lehrstuhl für Bergbaukunde - Fördertechnik und Konstruktionslehre, Department Mineral Resources Engineering, \\ Montanuniversität Leoben, Leoben, Österreich
}

Eingegangen 25. November 2020; angenommen 17. Januar 2021; online publiziert 4. Februar 2021

\begin{abstract}
Zusammenfassung: Viele Studierende arbeiten neben ihrem Studium - die einen müssen, um sich so ihre Ausbildung finanzieren zu können, die anderen, um einen Ausgleich zu schaffen und bereits erste Erfahrungen für ihren späteren Berufsweg zu erlangen. Während Gesprächen mit Freunden, Familie oder Mitmenschen kommt sehr oft die Frage "Was arbeitest du?" auf. Wenn die Antwort lautet "Ich arbeite in der Gastronomie." oder "Ich bin geringfügig im Handel tätig., haben die meisten Menschen einen Bezug und eine Vorstellung der Tätigkeit. Wird allerdings mit "Ich arbeite als studentische/r Mitarbeiterln an der Universität." geantwortet, wissen sehr viele nicht, welche Arbeitsbereiche umfasst werden. Dieser Beitrag gibt einen kurzen Überblick über die Aufgaben von studentischen MitarbeiterInnen der Arbeitsgruppe Fördertechnik und Konstruktionslehre des Lehrstuhls für Bergbaukunde, Bergtechnik und Bergwirtschaft.
\end{abstract}

Schlüsselwörter: Studentische MitarbeiterInnen, Diskrete Elemente Methode, Gurtfördertechnik,

Machbarkeitsstudien, Kontroll- und

Überwachungsarbeiten, Labore

\section{Assignments of Student Staff}

Abstract: Many students work additionally to their studies either to finance their education or to create a balance and to gain experience for their later career. During conversations with friends, family and others the question "What do you work?" comes up often. If the answer is "I work in catering." or "I work in sales.," most people have an idea of the job. If the answer is "I work as a student staff at the university.", it is mostly unknown which areas of work are

\footnotetext{
J. Leitner, BSc ( $₫)$

Montanuniversität Leoben,

Franz-Josef-Straße 18,

8700 Leoben, Österreich

jasmin.leitner@unileoben.ac.at
}

Lehrstuhl für Bergbaukunde - Fördertechnik und

Konstruktionslehre, Department Mineral Resources Engineering, included. This article gives a brief overview of the tasks of student staff from the working group Conveyor Technology and Design Methods at the Chair of Mining Engineering and Mineral Economics.

Keywords: Student staff, Discrete element method, Belt conveyor technology, Feasibility studies, Control and monitoring work, Laboratories

Im Zuge des Studiums an der Montanuniversität Leoben besteht für Studierende die Möglichkeit, bei den einzelnen Lehrstühlen als studentische Mitarbeiterlnnen zu arbeiten. Einer der Lehrstühle ist der Lehrstuhl für Bergbaukunde, Bergtechnik und Bergwirtschaft, zu dem auch die Arbeitsgruppe Fördertechnik und Konstruktionslehre gehört. Durch die Mitarbeit an verschiedenen Projekten mit Firmenpartnern lernen die studentischen Mitarbeiterlnnen unterschiedliche Bereiche kennen und bekommen dadurch einen guten Einblick in das spätere Arbeitsleben.

Ein großer Bereich der Arbeiten der studentischen MitarbeiterInnen ist die Simulation mit der Diskreten Elemente Methode (DEM). Mit dieser Simulation werden einerseits bereits bestehende Anlagen und andererseits Anlagen in der Entwicklungsphase nachgestellt, um die vorgegebenen Funktionen der Anlage zu testen und kontrollieren. Für die Kalibration der Simulation werden Parameter benötigt, welche durch Schüttgutversuche ermittelt werden. Es werden für verschiedene Firmenpartner Simulationen durchgeführt, wie beispielsweise die in Abb. 1 dargestellte Zentralschurre eines Schiffsbeladers der Firma Sandvik.

Der Lehrstuhl besitzt eine eigene Versuchsanlage, welche aus vier Förderbändern besteht, die das Schüttgut im Kreis transportieren. Bei dieser können unterschiedliche Prototypen der Gurtfördertechnik, Schurren im Modellmaßstab oder auch Schüttgut auf deren Verhalten getestet werden. Mithilfe der angebrachten Prüf- und Überwachungssystemen werden unterschiedliche Daten gemessen, welche mit der Simulation verglichen werden. Diese Versuchsanlage wurde von studentischen MitarbeiterInnen 


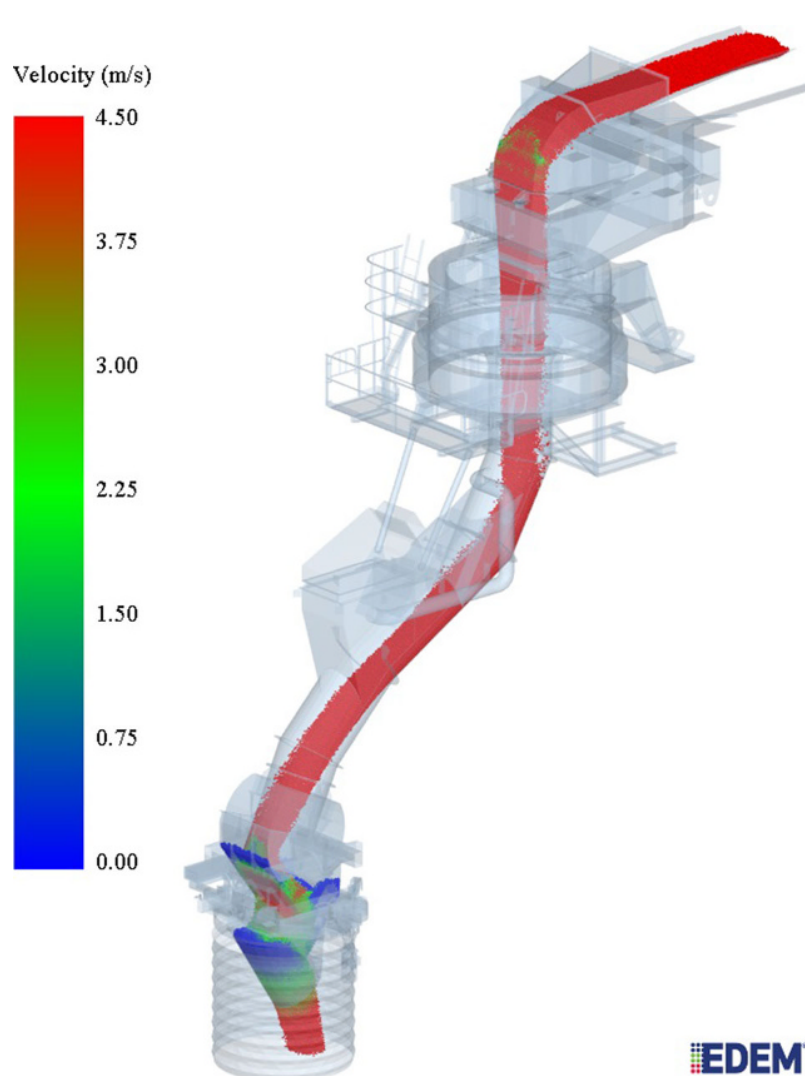

Abb. 1: Zentralschurre eines Schiffsbeladers

im Rahmen einer Bachelorarbeit konstruiert, ausgelegt und aufgebaut.

Im Zuge eines Projektauftrags mit der Firma REMA TIP TOP AG sollte ein bereits bestehendes Gurtdickenmessgerät verbessert beziehungsweise neu entwickelt werden. Für den Prototyp wurde in sehr kurzer Zeit ein Konzept erarbeitet und umgesetzt, um diesen bei der Bauma 2019 vorzustellen. Danach wurde der Prototyp weiterentwickelt und verbessert, um die gestellten Vorgaben zu erfüllen. All dies wurde von studentischen MitarbeiterInnen unter Absprache mit dem Firmenpartner und dem Betreuer realisiert. In Abb. 2 ist das entwickelte Gerät abgebildet.

Des Weiteren werden auch Machbarkeitsstudien durchgeführt, bei denen herausgefunden werden soll, ob und wie die vorgegebene Aufgabenstellung realisiert werden kann.

Ein anderer Aufgabenbereich sind die Kontroll- und Überwachungsarbeiten bei Projekten. Dabei wird beispiels- weise für einen Projektpartner eine Schurre konstruiert, auf die verschiedene Materialien aufgetragen werden, um diese auf Verschleiß zu testen. Die Aufgabe der studentischen MitarbeiterInnen ist dabei, die Schurre zu konstruieren, den Einbau zu kontrollieren und den Verschleiß der aufgetragenen Materialen zu kontrollieren und überwachen.

Für studentische MitarbeiterInnen besteht auch die Möglichkeit, in der lehrstuhleigenen Werkstatt zu arbeiten. Dort werden zum Beispiel selbst konstruierte Prüfstände gebaut, Schweißarbeiten, Reparaturen und Umbauarbeiten für die Versuchshalle, die Werkstatt und die diversen Prüfstände in den Laboren durchgeführt.

Zu dem stehen Labore zur Verfügung, in denen Schüttgutparameter ermittelt werden können. In diesen besteht auch die Möglichkeit mittels eines 3D-Druckers Prototypen zu bauen.

Für die einzelnen Projekte müssen Literaturrecherchen durchgeführt werden, wobei die studentischen MitarbeiterInnen sehr viele Erfahrungen sammeln, welche sie für ihre eigenen Bachelor- und Masterarbeiten nutzen können.

Funding. Open access funding provided by Montanuniversität Leoben.

Open Access Dieser Artikel wird unter der Creative Commons Namensnennung 4.0 International Lizenz veröffentlicht, welche die Nutzung, Vervielfältigung, Bearbeitung, Verbreitung und Wiedergabe in jeglichem Medium und Format erlaubt, sofern Sie den/die ursprünglichen Autor(en) und die Quelle ordnungsgemäß nennen, einen Link zur Creative Commons Lizenz beifügen und angeben, ob Änderungen vorgenommen wurden.

Die in diesem Artikel enthaltenen Bilder und sonstiges Drittmaterial unterliegen ebenfalls der genannten Creative Commons Lizenz, sofern sich aus der Abbildungslegende nichts anderes ergibt. Sofern das betreffende Material nicht unter der genannten Creative Commons Lizenz steht und die betreffende Handlung nicht nach gesetzlichen Vorschriften erlaubt ist, ist für die oben aufgeführten Weiterverwendungen des Materials die Einwilligung des jeweiligen Rechteinhabers einzuholen.

Weitere Details zur Lizenz entnehmen Sie bitte der Lizenzinformation auf http://creativecommons.org/licenses/by/4.0/deed.de.

Abb. 2: Gurtdickenmessgerät

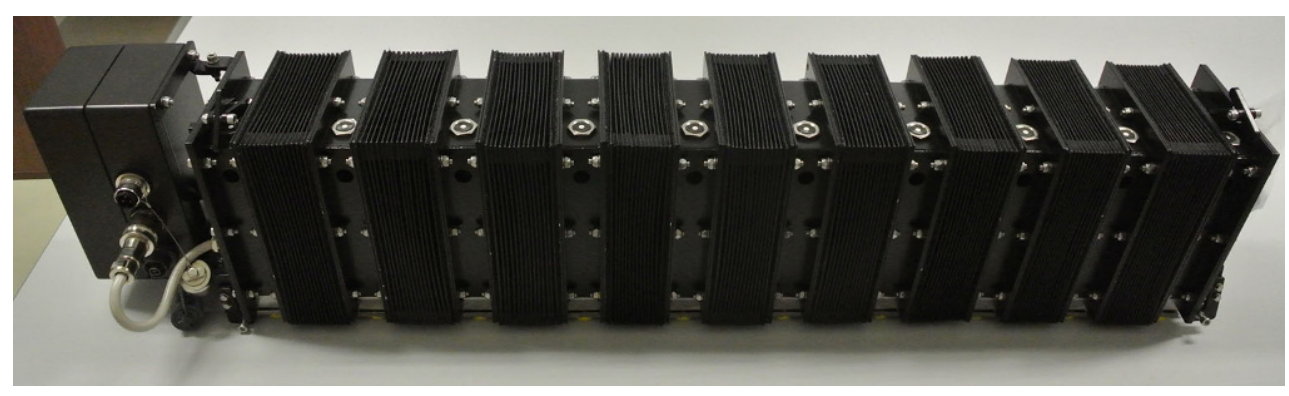

